\author{
С. ЛЕБЕДЕВА
}

\title{
СИСТЕМЫ МОДАЛЬНОГО ИСЧИСЛЕНИЯ ИМЁН
}

\author{
(Резюме)
}

Во второй части статьи оговорены две системы модального исчисления имён: система $O_{\mu}^{\prime}$ и система $O_{\mu}^{\prime \prime}$.

В языке этих систем выступают именные переменные, постоянные термины онтологии, а также модальные термины $\mu$ (может быть) и $\lambda$ (должно быть), являюшиеся порождающими имена функторами. Первый термин является первичным термином, второй есть термином определяемым следующим образом:

$$
x \lambda S \equiv x \varepsilon x \wedge \sim x \mu S^{\prime}
$$

Кроме аксиомы онтологии АО, аксиомами системы $O_{\mu}^{\prime}$ являются выражения:

A1. $x \mu S \equiv \sum_{y}(y \mu x) \wedge \prod_{\nu, z}(y \mu x \wedge z \mu x \rightarrow y \mu z) \wedge \prod_{y}(y \mu x \rightarrow y \mu S)$

A2. $x \varepsilon S \rightarrow x \mu S$

A3. $x \mu S+P \rightarrow x \mu S \vee x \mu P$

A4. $x \mu y \wedge \rightarrow|y| \rightarrow y \mu x$

A5. $x \mu S \wedge S a P \rightarrow x \mu P$

Правилами системы $O_{\mu}^{\prime}$ являются правила онтологии.

Аксиомами системы $O_{\mu}^{\prime \prime}$ являются выражения А0-А4. Правилами системы $O_{\mu}^{\prime \prime}$ являются правила онтологии а также

ПрАвилО I: Если выражение $x \varepsilon \omega_{1} \rightarrow x \varepsilon \omega_{2}$ есть доказуемым предложением онтологии, то выражение $x \mu \omega_{1} \rightarrow x \mu \omega_{2}$ также есть доказуемым предложением онтологии ( $\omega_{1}$ и $\omega_{2}-$ именные выражения).

Система $O_{\mu}^{\prime \prime}$ остро заключена в системе $O_{\mu}^{\prime}$, которая в свою очередь остро заключена в системе $O_{L}$. Системы $O_{\mu}^{\prime \prime}$ и $O_{S 5}$ пересекаются. В статье доказывается, что аксиома А5 не есть доказуемым предложением системы $O_{\mu}^{\prime \prime}$.

Автор статьи считает, что среди систем $O_{L}, O_{S 5}, O_{\mu}^{\prime}$ и $O_{\mu}^{\prime \prime}$ систем $O_{\mu}^{\prime \prime}$ наиболее близка интуициям связанным с употреблением модалыных терминов в разговорном языке. 